\title{
The neutrons for science facility at SPIRAL-2
}

X. Ledoux ${ }^{1, a}$, M. Aïche ${ }^{4}$, M. Avrigeanu ${ }^{10}$, V. Avrigeanu ${ }^{10}$, E. Balanzat ${ }^{14}$, B. Ban-d'Etat ${ }^{14}$, G. Ban ${ }^{5}$, E. Bauge ${ }^{3}$, G. Bélier ${ }^{3}$, P. Bém ${ }^{7}$, C. Borcea ${ }^{10}$, T. Caillaud ${ }^{3}$, A. Chatillon ${ }^{3}$, S. Czajkowski ${ }^{4}$, P. Dessagne ${ }^{6}$, D. Doré ${ }^{2}$, U. Fischer ${ }^{9}$, M.O. Frégeau $^{1}$, J. Grinyer ${ }^{1}$, S. Guillous ${ }^{14}$, F. Gunsing ${ }^{2}$, C. Gustavsson ${ }^{8}$, G. Henning ${ }^{6}$, B. Jacquot ${ }^{1}$, K. Jansson ${ }^{8}$, B. Jurado ${ }^{4}$, M. Kerveno ${ }^{6}$, A. Klix ${ }^{9}$, O. Landoas $^{3}$, F.R. Lecolley ${ }^{5}$, J.L. Lecouey ${ }^{5}$, M. Majerle ${ }^{7}$, N. Marie ${ }^{5}$, T. Materna ${ }^{2}$, J. Mrázek ${ }^{7}$, F. Negoita ${ }^{10}$, J. Novák ${ }^{7}$, S. Oberstedt ${ }^{11}$, A. Oberstedt ${ }^{15}$, S. Panebianco ${ }^{2}$, L. Perrot ${ }^{13}$, A.J.M. Plompen ${ }^{11}$, S. Pomp ${ }^{8}$, A.V. Prokofiev ${ }^{8}$, J.M. Ramillon ${ }^{14}$, F. Farget ${ }^{1}$, D. Ridikas ${ }^{2}$, B. Rossé $^{3}$, O. Sérot ${ }^{12}$, S.P. Simakov ${ }^{9}$, E. Šimečková ${ }^{7}$, M. Stefánik ${ }^{7}$, J.C. Sublet ${ }^{16}$, J. Taïeb ${ }^{3}$, D. Tarrío ${ }^{8}$, L. Tassan-Got ${ }^{13}$, I. Thfoin $^{3}$, and C. Varignon ${ }^{3}$

1 GANIL, CEA/DRF - CNRS/IN2P3, Boulevard Henri Becquerel, BP. 55027, 14076 Caen Cedex 5, France

2 Irfu, CEA, Université Paris-Saclay, 91191 Gif-sur-Yvette, France

CEA/DAM/DIF, 91297 Arpajon, France

4 CENBG, Université Bordeaux 1, CNRS/IN2P3, UMR 5797, Chemin du Solarium, BP. 120, 33175 Gradignan, France

5 LPC Caen, ENSICAEN, Université de Caen, CNRS/IN2P3, 14050 Caen Cedex, France

${ }^{6}$ Université de Strasbourg, CNRS, IPHC UMR 7178, 67000 Strasbourg, France

7 NPI, Řež, Czech Republic

8 Department of Physics and Astronomy, Uppsala University, Box 516, 75120 Uppsala, Sweden

9 KIT, Karlsruhe, Germany

NIPNE, Bucharest, Romania

11 EC-JRC Geel, Retieseweg 111, 2440, Belgium

12 CEA/DEN, Cadarache, 13108 Saint-Paul-lez-Durance, France

13 IPNO, CNRS-IN2P3, Université Paris-Sud, Université Paris-Saclay, 91406 Orsay Cedex, France

14 CIMAP, Boulevard Henri Becquerel, BP. 5133, 14070 Caen Cedex 5, France

15 ELI-NP, Bucharest-Magurele, Romania

16 Culham Centre for Fusion Energy, UK

\begin{abstract}
Numerous domains, in fundamental research as well as in applications, require the study of reactions induced by neutrons with energies from few $\mathrm{MeV}$ up to few tens of $\mathrm{MeV}$. Reliable measurements also are necessary to improve the evaluated databases used by nuclear transport codes. This energy range covers a large number of topics like transmutation of nuclear waste, design of future fission and fusion reactors, nuclear medicine or test and development of new detectors. A new facility called Neutrons For Science (NFS) is being built for this purpose on the GANIL site at Caen (France). NFS is composed of a pulsed neutron beam for time-of-flight facility as well as irradiation stations for cross-section measurements. Neutrons will be produced by the interaction of deuteron and proton beams, delivered by the SPIRAL-2 linear accelerator, with thick or thin converters made of beryllium or lithium. Continuous and quasi-mono-energetic spectra will be available at NFS up to $40 \mathrm{MeV}$. In this fast energy region, the neutron flux is expected to be up to 2 orders of magnitude higher than at other existing time-of-flight facilities. In addition, irradiation stations for neutron-, proton- and deuteron-induced reactions will allow performing cross-section measurements by the activation technique. After a description of the facility and its characteristics, the experiments to be performed in the short and medium term will be presented.
\end{abstract}

\section{Introduction}

The Neutrons For Science (NFS) facility is under construction on the GANIL [1] site at Caen (France). This facility will be dedicated to the study of neutron and light charged particle induced reactions. The energy range covered (up to $40 \mathrm{MeV}$ ) is of prime interest for fundamental research and applications. Among these topics we can mention the need of nuclear data for new reactor generation, the fusion technology or the medical applications. NFS is one of the components of SPIRAL-2 [2].

a e-mail: xavier.ledoux@ganil.fr
NFS will deliver continuous and quasi-mono-energetic neutron beams up to $40 \mathrm{MeV}$. The proton and deuteron beams accelerated by the linear accelerator are particularly well suited for production of high neutron flux. NFS is equipped with a long time-of-flight area designed for the study of $(\mathrm{n}$, fission), $(\mathrm{n}, \mathrm{xn})$ or $(\mathrm{n}, \mathrm{lcp})$ reactions in the fast energy range. An irradiation station will also be available for measurements by activation method of neutron and ion induced reactions. Some applications like the production of radio-isotopes for medical applications or electronic chip irradiations will also be investigated. NFS will open GANIL/SPIRAL-2 to a new community of physicists in academic research as well as in several fields of applications. 


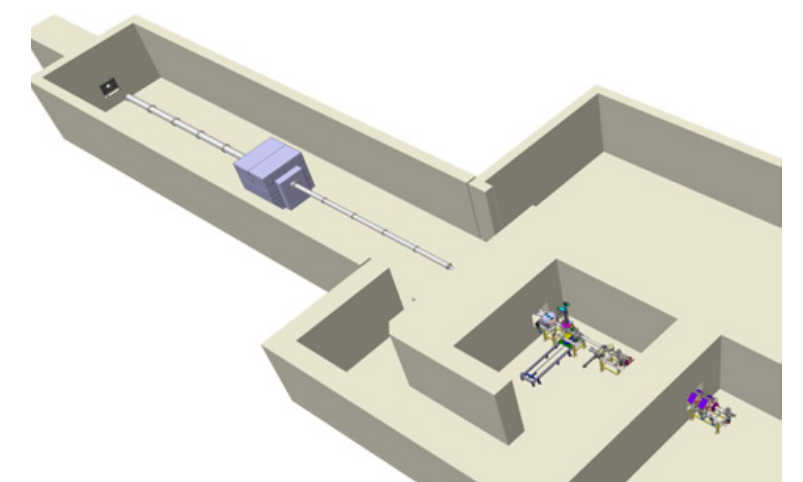

Figure 1. View of the 2 areas of NFS. The converter cave where neutrons are produced and the TOF area for experiments with the neutron beam.

Table 1. Energy/intensity range of beams accelerated by the LINAC.

\begin{tabular}{|l|c|c|c|c|}
\hline & $\mathbf{H}^{+}$ & ${ }^{2} \mathbf{H}^{1+}, \mathbf{H e}^{2+}$ & \multicolumn{2}{l|}{ Heavy ions } \\
\hline & $\mathrm{Q} / \mathrm{A}=1$ & $\mathrm{Q} / \mathrm{A}=1 / 2$ & $\mathrm{Q} / \mathrm{A} \geq 1 / 3$ & $\mathrm{Q} / \mathrm{A} \geq 1 / 6$ \\
\hline $\mathrm{Imax}(\mathrm{mA})$ & 5 & 5 & 1 & 1 \\
\hline $\mathrm{E}_{\max }$ & 33 & 20 & 14.5 & 8.5 \\
\hline
\end{tabular}

The SPIRAL-2 project is shortly described in Sect. 2. The NFS facility and its characteristics are presented in Sects. 3 and 4. The physics case and the first accepted experiments are developed in the Sect. 5. Finally the Sect. 6 gives a status of construction and a commissioning plan.

\section{SPIRAL-2}

\subsection{The project}

The SPIRAL-2 facility aims at extending significantly the current possibilities of Radioactive Ion Beam (RIB) physics and related applications. The production of high intensity RIB of neutron-rich nuclei will be based on fission of uranium target induced by neutrons, obtained from a deuteron beam impinging on a graphite converter (up to $10^{14}$ fissions/s). The post acceleration of RIB in the SPIRAL-2 project will be assured by the existing CIME cyclotron, which is well adapted for separation and acceleration of ions in the energy range from about 3 to $10 \mathrm{MeV} / \mathrm{u}$ for masses $\mathrm{A} \sim 100-150$.

In addition to the production of intense RIB, 3 experimental areas will be built around SPIRAL-2. The super spectrometer separator S3 [3], DESIR [4] and NFS. The project was split in 2 phases, the phase 1 includes $S 3$, NFS and DESIR. The production building for RIB based on fission target is part of the phase 2 and is not yet funded.

\subsection{The linear accelerator}

The SPIRAL-2 accelerator is a superconducting light/heavyion linear accelerator with an acceleration potential of about $40 \mathrm{MV}$. It will be able to deliver $5 \mathrm{~mA}$ of deuteron beam up to $40 \mathrm{MeV}$ and $1 \mathrm{~mA}$ of heavy ions beam up to 14.5 MeV/u. The beam characteristics are given in Table 1.

The first proton beam was extracted from the source in December 2014 and the first beam was accelerated by the RFQ in December 2015. The commissioning of the accelerator is ongoing and the first beam at the exit of the LINAC is expected for 2017 [5].

\section{The neutrons for science facility}

The facility is mainly composed of two underground experimental areas at $-9.5 \mathrm{~m}$, separated by a thick wall in which the collimator is installed. Another room at the level $-6 \mathrm{~m}$, hosts the electronic needed by the process as well as by some experimental set-ups. During data taking the physicists will operate from the acquisition room at ground level.

\subsection{The converter cave}

This room contains the beam line extension, connected to the accelerator, the converters to produce neutrons, the irradiation station and the pneumatic transfer system. Experiments using ion induced reactions will also be installed in this area. The beam line is composed of magnetic elements for beam driving, Faraday cups and beam profile monitors, a deflecting magnet and an ion beam dump.

\subsubsection{The converters}

NFS will be equipped with 2 converter devices.

Thin converters made of lithium or beryllium will be used to produce quasi-mono-energetic neutron beams up to $31 \mathrm{MeV}$ by using proton beam. The converter is fixed on a copper plate cooled by water. The magnet placed downstream of the converter deflects the crossing proton beam to the ion beam dump.

A thick converter $(8 \mathrm{~mm})$ will be used to ensure the production of neutrons with a continuous spectrum. The target consists of a rotating wheel of carbon or beryllium. This converter is designed to sustain a power deposition of $2 \mathrm{~kW}$, corresponding to a $50 \mu \mathrm{A}$ beam of $40 \mathrm{MeV}$ deuterons.

\subsubsection{The sample transfer system}

NFS will be equipped with a fast pneumatic transfer system to move sample from the converter room to the TOF area. After irradiation, sample will be moved quickly to a detection system allowing the activity measurement of isotopes with short half-life. Each sample is placed in a shuttle capsule moving inside a plastic pipe. For neutron irradiation the sample is put in air just downstream of the converter. This set-up will allow measuring reaction crosssections by activation technique.

\subsubsection{The irradiation station}

An irradiation station was built to perform cross-section measurements of ion induced reactions. It allows putting the sample under vacuum environment in order to be irradiated by charged particles. The irradiation station is equipped with a Faraday cup to monitor the ion beam and it is also connected to the pneumatic transfer system (see 3.1.2).

\subsection{The time-of-flight hall}

The time-of-flight hall, placed downstream of the converter room, is $28 \mathrm{~m}$ long and $6 \mathrm{~m}$ wide. The length of the room 
was chosen to perform energy measurements with a good resolution. Several experiments could be performed at the same time at different distances.

\subsubsection{The collimator}

The 2 rooms are separated by a thick $(3 \mathrm{~m})$ wall of concrete in which a collimator is installed. The collimator defines the neutron beam size along the TOF area thanks to a set of hollow cylinders put into an iron pipe. The tube is surrounded by concrete, iron, borated polyethylene and lead. The pieces in the central pipe have been designed (based on neutron transport simulations) to minimize the scattering of neutrons "outside of the beam envelope". The beam has a conical shape with an exit radius of $2 \mathrm{~cm}$ at 1 meter downstream of the collimator. Furthermore, the neutron beam profile can be modified for specific experiment needs. This is achieved by replacing the set of internal pieces of the collimator.

\subsubsection{The neutron beam dump}

The interaction of the neutron beam with the shielding wall at the end of the TOF area will produce neutrons (by backscattering) and photons (n,n' or n, $\gamma$ reactions), which will induce background. In order to minimize this effect a neutron beam dump was installed in a $4 \mathrm{~m}$ deep hole of square cross-section pierced in the concrete wall. The beam dump acts as a "neutron trap". It is composed of a lead wall, a tank filled with water and a polyethylene plate. The lead wall and the tank are pierced in their centre by a cylindrical hole whose diameter is larger than the beam spot diameter. The neutron beam enters in the beam dump, interacts with the polyethylene plate which minimizes the neutron backscattering. The water in the tank catches the main part of the backscattered neutrons. A large part of the photons are stopped by the $5 \mathrm{~cm}$ thick lead shield.

\subsubsection{The beam line and the second collimator}

The neutrons from the beam can be scattered by interacting with the nuclei in the air. This would generate background (neutron and gamma) potentially detrimental to the experiments. Thus a beam line under vacuum can be installed through the TOF hall. The neutron beam, having a conical shape, its size can be unsuitable to experiments placed at long distances. Hence a second collimator has been designed and will be installed in the middle of the TOF area in order to redefine the neutron beam. The central part of this disposal can be dismounted if needed in order to avoid additional background upstream of the second collimator.

\subsection{Beam monitoring}

A set of monitors will be available to measure the beam characteristics, energy distribution and neutron fluxes. The monitoring will be adapted to the experiment needs and will depend on the neutron beam characteristics: energy range, intensity, pulsed or not.

The first monitor is composed of a liquid scintillator (EJ309) coupled to a phototube. It allows measuring the neutron energy distribution by the TOF technique. The neutron gamma discrimination is performed by a pulse

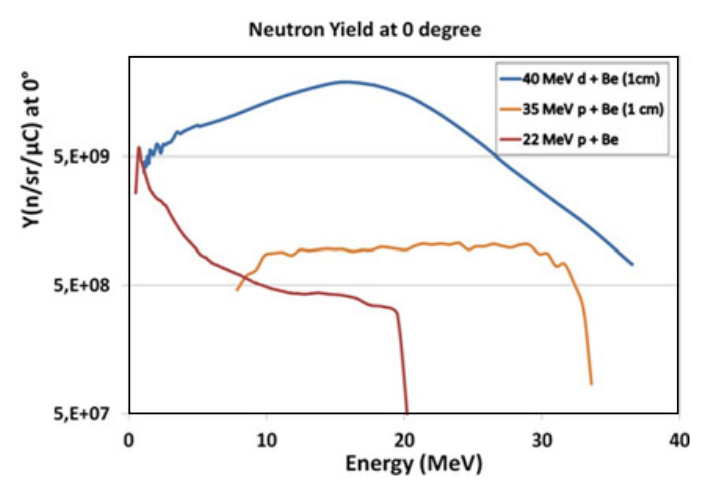

Figure 2. Neutron energy distribution from proton and deuteron + (thick) Be target (from Refs. 6 and 7). The measurement thresholds are different for the 3 set of data.

shape analysis. The scintillator is in a cylindrical cell with a diameter of $5 \mathrm{~cm}$ and a length of $7.5 \mathrm{~cm}$. This detector is quite efficient $(>10 \%)$ and will be used with a pulsed beam. The monitor characterisation (efficiency and light production) is under progress thanks to experiments with neutron beam.

A proton recoil spectrometer has been built for spectrum and flux measurement. It is composed of a thin polyethylene radiator and three silicon detectors to detect recoiling protons. It allows to measure the spectrum and the flux without any specific time structure. However the detection efficiency is very low $\left(<10^{-5}\right)$. The choice of the radiator thickness is a compromise between the energy resolution and the detection efficiency. Since it is almost impossible to cover the whole energy range up to $40 \mathrm{MeV}$ with a unique "radiator $\Delta \mathrm{E} 1-\Delta \mathrm{E} 2-\mathrm{E}$ " arrangement, the proton recoil spectrometer must be versatile to be adapted to the energy range of interest.

A Micromegas gas detector with a thin ${ }^{238} \mathrm{U}$ deposit will be used as relative monitor. The gas volume is separated in two regions by thin micromesh, the first one where the conversion and drift of the ionization electrons occur, and the second one, 50-160 micron thick, where the amplification takes place. Ionization electrons are created by the energy deposition of charged particle (fission fragments generated in the converted) in the conversion gap. Since the detector is very thin, it can be placed at the exit of the collimator without perturbing the experiments. ${ }^{238} \mathrm{U}$ was chosen as converter because its fission cross section presents a threshold around $0.7 \mathrm{MeV}$, making this detector insensitive to thermal neutrons.

\section{Beam characteristics}

\subsection{Energy spectra}

The neutron energy distribution depends on the reaction and on the target thickness.

A continuous energy spectrum is obtained using a thick beryllium target (thick enough to stop the beam). Both proton and deuteron beam can be used but deuterons produce much higher neutron yield as shown in Fig. 2. With maximal deuteron energy at 0 degree, the spectrum extends up to $40 \mathrm{MeV}$ with an average value of approximately $14 \mathrm{MeV}$. When the beam is fully stopped in the target, the dissipated power can reach $2 \mathrm{~kW}$ at incident beam intensity of $50 \mu \mathrm{A}$. 


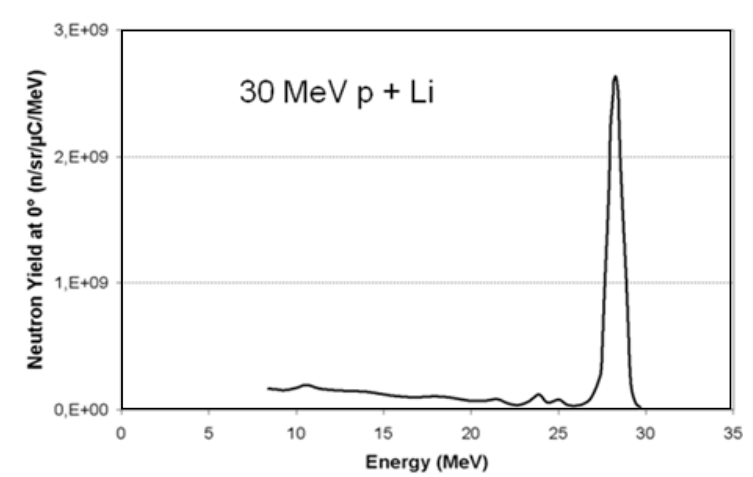

Figure 3. Neutron energy distribution produced by the $30 \mathrm{MeV}$ $\mathrm{p}+\mathrm{Li}$ reaction (from Ref. 8).

Neutrons can also be generated by ${ }^{7} \operatorname{Li}(\mathrm{p}, \mathrm{n}){ }^{7} \mathrm{Be}$ or ${ }^{9} \mathrm{Be}(\mathrm{p}, \mathrm{n}){ }^{9} \mathrm{~B}$ reactions. ${ }^{7} \mathrm{Li}(\mathrm{p}, \mathrm{n}){ }^{7} \mathrm{Be}$ reaction on a thin lithium converter produces close to mono-energetic neutrons as shown in Fig. 3. The neutron spectrum at $0{ }^{\circ}$ Consists of a mono-energetic peak due to the ${ }^{7} \mathrm{Li}(\mathrm{p}, \mathrm{n})$ process and a continuum which is attributed to the break-up process. The protons after crossing the lithium converter are swept out of the neutron beam thanks to a bending magnet placed downstream of the target.

\subsection{Time structure}

In most of the cases a pulsed beam is request to measure the neutron energy. The beam frequency must be adapted to the neutron energy range and to the flight path. At NFS the flight path extends from 5 to $30 \mathrm{~m}$ and the energy domain of interest from 0.1 up to $40 \mathrm{MeV}$. To avoid the overlap of a $0.1 \mathrm{MeV}$ neutrons from one burst with a $40 \mathrm{MeV}$ neutron from the next one, the beam period must be larger than $\approx 1 \mu \mathrm{s}$ (corresponding frequency should be below $1 \mathrm{MHz}$ ) for a flight path of $5 \mathrm{~m}$. The own LINAG frequency $(88 \mathrm{MHz})$ is not suitable therefore a fast chopper is added to decrease the frequency by a factor of 100 or more.

\subsection{Flux}

The neutron fluxes have been estimated from the production yield, the beam intensity and the distance from the source. For radioprotection point of view the design case is a $50 \mu \mathrm{A}$ of deuteron beam at $40 \mathrm{MeV}$ impinging a thick beryllium converter.

For irradiation purposes at $10 \mathrm{~cm}$ downstream of the converter, the integrated flux will be of the order of $10^{11} \mathrm{n} \cdot \mathrm{s}^{-1} \cdot \mathrm{cm}^{-2}$ (maximum value of $10^{11} \mathrm{n} \cdot \mathrm{s}^{-1} \cdot \mathrm{cm}^{-2}$. $\mathrm{MeV}^{-1}$ around $14 \mathrm{MeV}$ ). In the time of flight area, at $5 \mathrm{~m}$ from the converter, the flux will be close to $10^{8} \mathrm{n} \cdot \mathrm{s}^{-1} \cdot \mathrm{cm}^{-2}$. The comparison with other existing TOF facilities worldwide is presented in Fig. 4. It is seen that between a few $\mathrm{MeV}$ and $30 \mathrm{MeV}$, the average flux is up to two orders of magnitude higher than the one available at other existing facilities, mainly due to the high repetition rate of the beam. Contrary to the other facilities based on spallation reaction or photo-fission NFS does not cover the low and high energy region and also the instantaneous flux at NFS will be quite low.

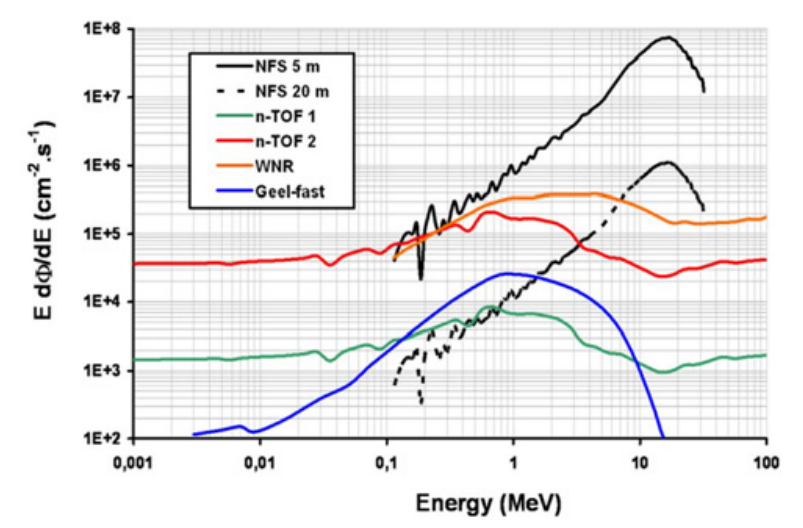

Figure 4. Neutron flux and energy spectra at several Time-offlight facilities. The black lines represent the NFS neutron fluxes at 5 and $20 \mathrm{~m}$ flight-path.

\section{Physics case}

\subsection{Letters of intent}

The NFS physics case includes fundamental physics and applications. The study of reactions induced by neutron and by light charged particle between few hundred of $\mathrm{keV}$ and $40 \mathrm{MeV}$ represents a large part of the foreseen experiments. NFS will also be an important tool for applications in biology and irradiation of electronic devices. Indeed there is a strong demand in experimental capabilities for neutron irradiation in the fast energy regime.

Since 2011, 19 letters of intent (LOI) have been submitted to the GANIL/SPIRAL-2 scientific advisory committee. Several sub-topics can be distinguished like the study of $(n, x n)$ reactions, the production of light charged particles, the development of detectors and mainly the study of the fission process.

\subsection{Proposals for first experiments}

In June 2016, for the first time the GANIL PAC (proposal advisory committee) opened the call for NFS experiments. The beam offer was limited for technical reasons related to the commissioning phase of the accelerator. Only proton and alpha beams at $88 \mathrm{MHz}$ were proposed. This made the use of TOF technique almost impossible and consequently limited the possible experiments. Nevertheless, in total ten experiments were proposed. A short description of the some of these accepted experiments is given below.

- The PFNS (prompt fission neutrons spectra) in ${ }^{238} \mathrm{U}$ neutron induced fission will be measured at $6.5 \mathrm{MeV}$ just below the $2^{\text {nd }}$ chance of fission. At this energy the neutron emission by the compound nucleus before it fissions is important and still need to be characterized. The experimental set-up is composed of a fission chamber [9], used also as a target, and an array of 50 neutron detectors based on liquid scintillator with neutron gamma discrimination capabilities.

- The ${ }^{238} \mathrm{U}(\mathrm{n}, 2 \mathrm{n})$ and ${ }^{238} \mathrm{U}(\mathrm{n}, 3 \mathrm{n})$ reaction cross-sections will be measured from the reaction threshold up to $25 \mathrm{MeV}$. It is a preliminary experiment to the measurement of ${ }^{239} \mathrm{Pu}(\mathrm{n}, \mathrm{xn})$ reaction cross-sections where data disagree in the threshold energy region. The technique consists of using a fission chamber as 
an active target and a $4 \pi$ neutron detector. It is a quite challenging experiment since the neutrons emitted in the $(n, x n)$ reactions have to be separated from fission neutrons. For that purpose the fission chamber has to be a very efficient veto to discriminate fission events.

- The double differential cross-section of light charged particle emission in neutron induced reaction on carbon will be measured. These data are of prime interest for applications like the damage characterization for electronics, the nuclear energy or the medical applications. These reactions lead to the production of highly ionizing particles (mainly protons or alphas). It induces damages on structural materials, generates gas or produces single event upsets in electronics. For these reasons reliable data are needed. The experiment will be performed with the MEDLEY detector [10,11] which is made of eight telescopes composed of two silicon detectors and one CsI.

- The direct measurement of the ${ }^{28} \mathrm{Si}(\mathrm{p}, \gamma)^{29} \mathrm{P}$ and ${ }^{29} \mathrm{Si}(\mathrm{p}, \gamma){ }^{30} \mathrm{P}$ reaction rates at $0.73 \mathrm{MeV}$ was proposed. These reactions play an important role in the hot CNO (Carbon, Nitrogen, Oxygen) cycle and a precise measurement is request. The goal is to improve significantly the uncertainty, which should allow to better constrain the nova models $\left({ }^{29} \mathrm{Si} /{ }^{28} \mathrm{Si}\right.$ and ${ }^{30} \mathrm{Si} /{ }^{28} \mathrm{Si}$ ratios in particular) and thus to understand the origin of presolar grains (from the comparison of new results from the model with the observations). The very high intensity of the Linac beam is an important advantage for such experiments.

- Measurement of the excitation function of proton induced reaction on iron is another accepted experiment. The activation technique will be used thanks to the irradiation station and the pneumatic transfer system available. Several short half lived isotopes $\left({ }^{53 \mathrm{~m}} \mathrm{Fe},{ }^{54 \mathrm{~m}} \mathrm{Co},{ }^{50 \mathrm{~m}} \mathrm{Mn},{ }^{52 \mathrm{~m}} \mathrm{Fe}\right)$ will be measured, what also includes separate measurements of ${ }^{58 \mathrm{~m}} \mathrm{Co}$ and ${ }^{58 \mathrm{~g}}$ Co production cross-sections.

- The production of radioisotopes for medical applications using alpha induced reactions will also be investigated. The possibility of tuning the optimal beam energy and therefore optimizing production rates is a strong point of NFS contrary to several cyclotrons where only fixed energies can be delivered. Actually in some cases the energy is a compromise between an optimized production rate and a low rate of contaminants.

- NFS will also be used for determining the neutron detection efficiency of the CsI detectors of the FAZIA detector. FAZIA [12] is a new $4 \pi$ charged particle detector composed of Si-Si-CsI telescopes. The last CsI stage is clearly sensitive to neutrons and its efficiency measurement is of prime interest to determine if neutrons detection is possible with this set-up.

\section{Status}

\subsection{Construction plan}

The SPIRAL-2 building is finished. The sources and the RFQ of the Linear accelerator are under commissioning with proton and alpha beams [5].
On the NFS part, the beam line in the converter room is almost installed as well as the thin lithium converter. The collimator between the two rooms is also installed. The neutron beam dump will be delivered soon. The rotating converter is undergoing laboratory tests. The irradiation station and the pneumatic transfer system will be installed at NFS by the end of 2016. It is expected that the facility will be fully completed by the end of 2016. The commissioning will start as soon as the beam can be delivered by the Linac.

\subsection{Commissioning plans}

A plan for NFS commissioning and first experiments was established taking into account the accelerator commissioning. The strategy is to alternate commissioning periods and experiments. The first experiment will probably be activation measurement with proton beam. Actually these experiments require low intensity and no time structure. Secondly, quasi-mono-energetic neutrons will be produced by ${ }^{7} \mathrm{Li}(\mathrm{p}, \mathrm{n})$ reaction at high energy $(\approx 30 \mathrm{MeV})$. The beam characterization will be performed before the first experiments. Later, lower energy neutron beams will be developed. The production of neutrons with a thick target will follow using a proton beam. When available, the alpha beam will be used for activation measurement. Finally, the deuteron beam will be tested and used at the very end of commissioning process.

\section{Conclusion}

NFS will be the first experimental area operational at SPIRAL-2 facility. It will be a very powerful tool for nuclear physics and applications. The neutron energy range $(0.1-40 \mathrm{MeV})$ and the time-of-flight area are particularly well suited for the study of (n,fission), (n,xn) or $(\mathrm{n}, \mathrm{lcp})$ reactions which are of prime interest for the development of reactors of new generation or the fusion technology. The intense neutron flux and the large energy spectrum range centred around $14 \mathrm{MeV}$ are also strong points for applications like fusion and also electronics irradiation.

In addition, NFS will deliver proton, deuteron and ion beams. They will be used for studies related to nuclear astrophysics or fusion technology development.

The neutron and ion beams characteristics are also suitable for the search of alternative production routes of radioisotopes for medical applications.

The first beam delivery to the target area is expected by 2017. A number of experiments have already been proposed by the international community and accepted by the GANIL Proposal Advisory Committee.

\section{References}

[1] GANIL, www.ganil-spiral2.eu

[2] M. Lewitowicz, "The SPIRAL2 Project: Physics and Challenges", Zakopane Conference on Nuclear Physics, September 2008

[3] A. Drouart, J. Nolen and H. Savajols, Int. J. of Modern Phys. E-Nuclear Physics 18(10), 2160 (2009)

[4] The DESIR facility at SPIRAL-2, Proceedings of the French-Japanese Symposium on Nuclear Structure 
Problems, January 5-8, 2011, RIKEN, Japan, World Scientific, ISBN: 978-981-4417-94-5, p. 224

[5] R. Ferdinand et al. "STATUS OF SPIRAL2 AND RFQ BEAM COMMISSIONING", In Proc. LINAC 2016, WE1A06, East Lansing (to be published) [6] M.J. Saltmarsh et al., NIMA 145, 81-90 (1977)
[7] H.J. Brede et al., NIMA 274, 332-344 (1989)

[8] C.J. Batty et al., NIM 68, 273-276 (1969)

[9] J. Taïeb et al., NIMA 833, 1-7 (2016)

[10] D. Tarrío, this conference

[11] K. Jansson et al., NIMA 794, 141 (2015)

[12] R. Bougault et al., EPJA 50, 1 (2014) 